\title{
The Fourier Notation of the Geomagnetic Signals Informative Parameters
}

\author{
Osvaldo Faggioni ${ }^{1,2}$ \\ ${ }^{1}$ DITEN-SEALab-ACM Laboratory, Department of Electric, Electronic, Telecommunication Engineering \\ and Naval Architecture, University of Genova, Genova, Italy \\ ${ }^{2}$ Istituto Nazionale di Geofisica e Vulcanologia, Rome, Italy \\ Email: osvaldo.faggioni@unige.it, osvaldo.faggioni@ingv.it
}

How to cite this paper: Faggioni, O. (2018) The Fourier Notation of the Geomagnetic Signals Informative Parameters. Journal of Signal and Information Processing, 9, 153-166.

https://doi.org/10.4236/jsip.2018.93009

Received: December 18, 2017

Accepted: August 4, 2018

Published: August 7, 2018

Copyright $\odot 2018$ by author and Scientific Research Publishing Inc. This work is licensed under the Creative Commons Attribution-NonCommercial International License (CC BY-NC 4.0). http://creativecommons.org/licenses/by-nc/4.0/

\begin{abstract}
The paper discusses the quantitative definition of the $\mathrm{s} / \mathrm{n}$ (signal to noise ratio) by means of new computational parameters derived (and computed) by the Fourier analysis. The theme is of great relevance when the geomagnetic observed field has high transient noise and high energy content (i.e. geomagnetic signal interfered by human activity magnetic band) and when the signal analysis action is oriented to the detection of magnetic sources characterized by quasi-punctiform size, low energy level and kinetic mechanical status (i.e. uw armed terrorist). The paper shows the results obtained introducing two new informative spectral parameters: the informative capability " $C$ " and the enhanced informative capability " $e C$ ". These parameters are depending on the comparison of the energy of the target signal with total field energy and they are characteristics of each elementary signal. $C$ classifies the energy of the spectrum in two metrological bands: elementary signal informative energy $E_{\mathrm{I}}$ (band or single signal) and passive energy $E_{p}$. This metrological classification of the energy overtakes the concept of noise: each signal is part of the noise band when it is not under observation and becomes out of the band when it is under observation (numerical observation $\rightarrow$ computation). $C$ (and $e C$ ) allows to compute the value of the "visibility" of the informative signals in a high energy geomagnetic field (or spectrum). $C$ is a fundamental parameter for the evaluation of the effectiveness of singularity magnetic metrology in the passive detection of small magnetic sources in high noised magnetic field.
\end{abstract}

\section{Keywords}

Geomatic, Informative Signal Analysis, Fourier Analysis, Geomagnetism, Metrology of Singularity, Frequency Domain Observations, S/N Manipulation 


\section{Introduction}

The passive magnetic detection effectiveness is depending on the amplitude of the target signal and on its geometric recognizability in time and frequency (spectral "visibility") domains. In the studies of low-frequency signals as for example modeling of the terrestrial crust [1] this approach can also provide very effective results. This is because these signals are well localized in the spectrum, are generally over-sampled and come from large static sources. The certain and invariant on time spectral localization associated with enough high amplitude is the basis of the visibility of each single signal expressed in a high noise field. The problem of the visibility becomes quite complex when the target signal is coming from a quasi-punctiform and low amplitude source, inside a high energy (noise) geomagnetic volume. In the other hand, the low visibility problem becomes practically unsolvable when this class of sources is moving and their signals are recorded by a fixed sensor. The measure action moves the independent variable from the space domain to the time domain making unpredictable on the spectrum the target signal localization. On time domain the target signal measured $\Lambda(t)$ is depending on the speed value (unknown and variable) of the target source [2]. The variation of the source's speed induces the metrological phenomenon of the variation of the measured $\Lambda(t)$ and the numerical phenomenon of the $N$ shift ( $N$ spectral order number) in the Fourier Series computation. In order to quantify the phenomena of the visibility and spectral shift of $N$, the " $\mathrm{C}$ " parameter "signal informative content" and its derivate " $e C$ " "enhanced signal informative content" were used. $C$ and $e C$ link the energy of each elementary signals (or band) to total magnetic field. The parameter " $C$ " (and $e \mathrm{C}$ ) allows to quantify the target signal's visibility concept. These factors allow the quantitative evaluation of target signal informative capability inside its own spectrum. These parameters enable also to evaluate:

1) The level of the increase of the target signal informative capability dues to robustness manipulation in frequency domain and;

2) The informative capability of the geomagnetic singularity metrology also in hard noise environment.

The numerical parameters $C$ and $e C$ allow to classify each signal with respect to its energy. They are also a function of the (measured) wavelength which in turn depends on the speed of the generating source. In theory they therefore allow to control the evolution in time of the signal of a moving source. The control of these parameters is one of the basic concepts of the geomagnetic metrology of singularity [3] and solves the problem of the capability to detect low amplitude magnetic signals generated by quasi-punctiform kinetic sources. This problem cannot be solved objectively by means of the standard signal detection and interpretation techniques and their related standard procedures of frequency filtering procedures.

\section{The Metrological Manipulation of the Energy Definition}

The energy E content in the geomagnetic field may be considered as sum of sev- 
eral elementary contributes coming from natural and man-made sources. The Earth magnetic field "Emf" can be classified in "natural band signals nb" and "man-made signal band mmb" [4]. Analytical observation of geomagnetic field of the urban areas, industrial and port facilities, railways, electric power line, etc... shows the $\mathrm{nb}$ and $\mathrm{mmb}$ can be overlapped [4]. This phenomenon (interference) is typical for high frequency band of nb [5] and low frequency band of $\mathrm{mmb}$. At $\mathrm{nb}$ and $\mathrm{mmb}$ is summomg a third band of geomagnetic signals: the induced band "ib". This band is very interesting in the detection actions because it contents also the target signal frequencies. ib is originated by the inductive action of $\mathrm{nb}$ and $\mathrm{mmb}$ on the natural (planetary) or man-made conductors. ib, for its physics characteristics, has very large spectrum. We observe the first problem of the passive magnetic detection action is the overlaps to $\mathrm{nb}$ and $\mathrm{mmb}$ (including hypothetical target signals) and ib.

$$
F=\mathrm{nb}+\mathrm{mmb}+\mathrm{ib}
$$

The $F$ revealed to the ground level (or underwater) cannot be modeled and its analytic study is, essentially, a metrologic one. In order to the detect action (association of generated signal-generating source) the energy $E$ of the geomagnetic field can be considered built by two components: an informative component ( $E_{I}$ Informative Energy) and a non informative one ( $E_{P}$ Passive Energy). $E_{I}$ is the energy associated to the target's signal (or band). $E_{p}$ is the energy of the all other signals detected. It does not carry information about the target and interferes with detection of $E_{I^{r}}$

$$
E=E_{I}+E_{P}
$$

Def 1: $E_{I}$ (Informative Energy) is the target signal (or target signals) energy. It is a quantitative parameter of the target signal (or target signals) visibility.

Def 2: $E_{P}$ (Passive Energy) is the energy associated to the not target signals. It is an inverse quantitative parameter of the target signal (or target signals) visibility.

Elementary signals energy computation, in fast and approximate form, may be performed by the technique "T.App." (Triangular Approx) (Figure 1). T.App.

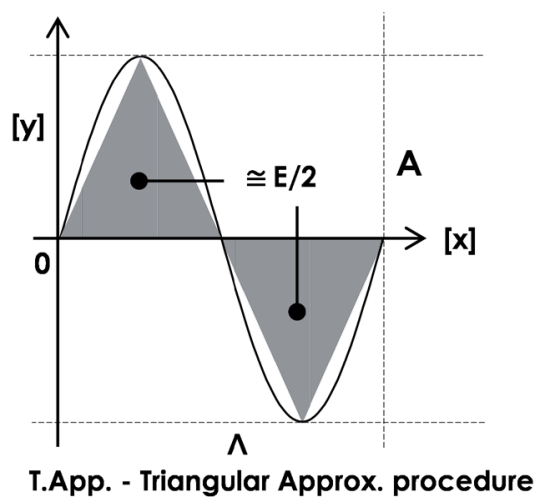

Figure 1. Triangular approximation of the energy $\mathrm{E}$ associated to a amplitude $A$ and wavelength $\Lambda$ signal. It has sufficient accuracy for the detection action (small kinetic sources in noised mag environmental). 
performs a good approximation for our work (counter-terrorism geomagnetic detection/protection), it is not heavy for the detection system intelligence and its TLC intranet protocols.

$$
\begin{gathered}
E_{I}^{(i)}=\frac{1}{4} A_{i} \Lambda_{i} \\
E_{P}^{(i)}=\frac{1}{4}\left(\sum_{i=1}^{n} A_{i} \Lambda_{i}-A_{i} \Lambda_{i}\right)
\end{gathered}
$$

from which (2)

$$
E=\frac{1}{4} \sum_{i=1}^{n} A_{i} \Lambda_{i}
$$

where

$A_{i}=$ amplitude of the $i$-th elementary harmonic $\Lambda_{i}=$ wavelength of the $i$-th elementary component harmonic.

Def 1 and Def 2 overcomes the noise concept: the energy of each signal is included in $E_{I}$ band when it is the object of the measure while its energy go on the $E_{P}$ band when it is not object of the measure. The metrology of singularity [3] defines the class of the signals [3]: each signal is not (or is) a component of the noise band $\left(E_{P}\right)$ if it is the object (or not) of the measure. From this point of view the signals of the earth's magnetic field are not classified by their geometrical and physical characteristics $(A, \Lambda, \Phi)$ but by their singularity. We observe that the measure of singularity also excludes all physical information about the Earth's magnetic field which however are not useful (indeed harmful) for detection action.

\section{The Digital Computation of the $E_{I}$ and $E_{P}$ Energy Bands}

The operative effectiveness of definitions 1 and 2 is linked to the capability to compute the numerical value of the $E_{i} \mathrm{~S}$ of the signals included in the geomagnetic field recording [6]. A good solution to obtain this one objective is the use of elementary signals $A_{i}$ (amplitude) and $\Lambda_{i}$ (wavelength) parameters use. $A_{i}$ and $\Lambda_{i}$ parameters are coming from the spectral composition of the Fourier harmonic parameters [7] " $a_{i}$ " so-called cos component or real component and " $b_{i}$ " so-called sin component or imaginary component [8] [9]:

Give the Fourier Series in trigonometric and periodical notation (period T)

$$
f(t)=\frac{1}{2} a_{0}+\sum_{i=1}^{n}\left(a_{i} \cos \omega_{i} t+b_{i} \sin \omega_{i} t\right)
$$

where $\omega_{i}$ is the pulsation

$$
\omega_{i}=\frac{2 \pi i}{T}
$$

and $a_{\mathrm{i}}$ and $b_{i}$ the coefficients of Fourier harmonic expansion

$$
a_{i}=\frac{2}{T} \int_{-T / 2}^{T / 2} f(t) \cos \omega_{i} t \mathrm{~d} t, i=0,1, \cdots
$$




$$
b_{i}=\frac{2}{T} \int_{-T / 2}^{T / 2} f(t) \sin \omega_{i} t \mathrm{~d} t, \quad i=1,2, \cdots
$$

the amplitude and wavelength of the $i$-th signal are computed by spectral analysis

$$
\begin{gathered}
A_{i}=\left(a_{i}^{2}+b_{i}^{2}\right)^{1 / 2} \\
\Lambda_{i}=\frac{T}{N_{i}}
\end{gathered}
$$

where $N_{i}$ is the Fourier harmonic expansion wave-number (computational number order N.O. or N).

The Equations (3) and (4) in expanded notation became

$$
\begin{gathered}
E_{I}^{(i)}=\frac{A_{i} \Lambda_{i}}{4}=\frac{T}{4} \frac{\left(a_{i}^{2}+b_{i}^{2}\right)^{1 / 2}}{N_{i}} \\
E_{P}^{(i)}=\frac{1}{4}\left(\sum_{i=1}^{n} A_{i} \Lambda_{i}-A_{i} \Lambda_{i}\right)=\frac{T}{4}\left[\sum_{i=1}^{n} \frac{\left(a_{i}^{2}+b_{i}^{2}\right)^{1 / 2}}{N_{i}}-\frac{\left(a_{i}^{2}+b_{i}^{2}\right)^{1 / 2}}{N_{i}}\right]
\end{gathered}
$$

We introduce now the parameter " $R$ relative amplitude" coming from the composition of $A(10)$ and $N$.

$$
R_{i}=\frac{\left(a_{i}^{2}+b_{i}^{2}\right)^{1 / 2}}{N_{i}}
$$

From which

$$
\begin{gathered}
E_{I}^{(i)}=\frac{T}{4} R_{i} \\
E_{P}^{(i)}=\frac{T}{4}\left[\sum_{i=1}^{n} R_{i}-R_{i}\right]
\end{gathered}
$$

Remembering: power $W$ to be equal $A^{2}(10)$.

$$
W=A^{2}=\left(a^{2}+b^{2}\right)
$$

Similarly to (14) we also define the "Jrelative power" to be:

$$
J_{i}=\frac{\left(a_{i}^{2}+b_{i}^{2}\right)}{N_{i}}
$$

The $E_{I}^{(i)}$ (12) expressed as a function of the respective order numbers $N_{i}$ is the "informative spectrum".

\section{The Digital Informative Parameters}

The acquisition of the physic information about the source from its signal is a typical metrological problem (also called inverse problem) [10]. It depends on the ability of the measuring system to read the signal. In order to obtain a quantitative (and objective) method of extracting the information from the signal we introduce three parameters of computation: the total informative content " $Q$ " (observed field); the elementary signal informative content " $C$ "; and the " $e C$ " 
elementary signal enhanced informative content (the definition of $e C$ is valid only for non-monochromatic signals).

Similarly to the (12) and (13) the (3) expressed in $R$ becomes:

$$
E=\frac{1}{4} \sum_{i=1}^{n} A_{i} \Lambda_{i}=\frac{T}{4} \sum_{i=1}^{n} \frac{\left(a_{i}^{2}+b_{i}^{2}\right)^{1 / 2}}{N_{i}}=\frac{T}{4} \sum_{i=1}^{n} R_{i}
$$

We compute the numerical values of $Q, C, e C$, as a function of $R$ with reference to their energy bands (by means of the harmonic development Fourier coefficients).

$$
\begin{gathered}
Q=E=\sum_{i=1}^{n} I E_{i}=\frac{1}{4} \sum_{i=1}^{n} A_{i} \Lambda_{i}=\frac{T}{4} \sum_{i=1}^{n} \frac{\left(a_{i}^{2}+b_{i}^{2}\right)^{1 / 2}}{N_{i}}=\frac{T}{4} \sum_{i=1}^{n} R_{i} \\
C_{i}=\frac{\frac{\left(a_{i}^{2}+b_{i}^{2}\right)^{1 / 2}}{N_{i}}}{\sum_{i=1}^{n} \frac{\left(a_{i}^{2}+b_{i}^{2}\right)^{1 / 2}}{N_{i}}}=\frac{R_{i}}{\sum_{i=1}^{n} R_{i}} \\
e C_{i}=\frac{\left(a_{i}^{2}+b_{i}^{2}\right)^{1 / 2}}{N_{i}} \\
\sum_{i=1}^{n} \frac{\left(a_{i}^{2}+b_{i}^{2}\right)^{1 / 2}}{N_{i}}-\frac{\left(a_{i}^{2}+b_{i}^{2}\right)^{1 / 2}}{N_{i}}=\frac{R_{i}}{\sum_{i=1}^{n} R_{i}-R_{i}}
\end{gathered}
$$

(21) and (22) are respectively, in the expanded form, useful for the production of the $C_{i}$ and $e C_{i}$ calculation algorithms (8) (9) (21) (22):

$$
C_{i}=\frac{\left[\left(\frac{2}{T} \int_{-T / 2}^{T / 2} f(t) \cos \omega_{i} t \mathrm{~d} t\right)^{2}+\left(\frac{2}{T} \int_{-T / 2}^{T / 2} f(t) \sin \omega_{i} t \mathrm{~d} t\right)^{2}\right]^{1 / 2}}{N_{i}}
$$

$$
\begin{aligned}
& \underline{\left[\left(\frac{2}{T} \int_{-T / 2}^{T / 2} f(t) \cos \omega_{i} t \mathrm{~d} t\right)^{2}+\left(\frac{2}{T} \int_{-T / 2}^{T / 2} f(t) \sin \omega_{i} t \mathrm{~d} t\right)^{2}\right]^{1 / 2}} \\
& e C_{i}=\frac{N_{i}}{\sum_{i=1}^{n} \frac{\left[\left(\frac{2}{T} \int_{-T / 2}^{T / 2} f(t) \cos \omega_{i} t \mathrm{~d} t\right)^{2}+\left(\frac{2}{T} \int_{-T / 2}^{T / 2} f(t) \sin \omega_{i} \mathrm{td} t\right)^{2}\right]^{1 / 2}}{N_{i}}-\frac{\left[\left(\frac{2}{T} \int_{-T / 2}^{T / 2} f(t) \cos \omega_{i} t \mathrm{~d} t\right)^{2}+\left(\frac{2}{T} \int_{-T / 2}^{T / 2} f(t) \sin \omega_{i} t \mathrm{~d} t\right)^{2}\right]^{1 / 2}}{N_{i}}}
\end{aligned}
$$

An useful analytical tool is the informative capability spectrum (Figure 2). As the same as the standard Fourier spectra (Amplitude $A$, Power $P$ and phase $\Phi$ ), we compose the $i$-th values of $C_{i}$ ( $Y$ variable) with the matching $N_{i}$ ( $X$ variable) (Figure 2). An interesting practical use of the information capacity spectrum $C$ is the variation of the parameter $C$ by varying the length of the reading window 
measure action

F(t) $\leftarrow--$ data

\section{Fourier analysis}

FFT

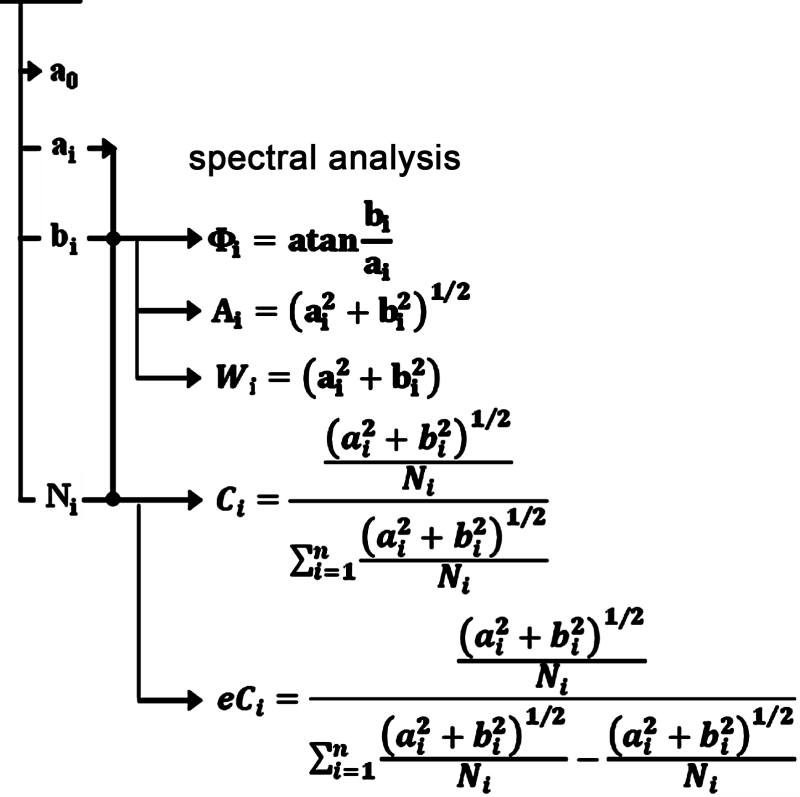

Figure 2. Algorithm to computation $C_{i}$ and e $C_{i}$ parameters in digital form from Fourier Serie (spectral flux of computation from data acquisition).

in which $C$ is represented [11]. This aspect concerns the choice of the operative window length $L$; the optimization $L$ depends on two opposite parameters:

1) by decreasing $\mathrm{L}$, the risk of corruption of the information increases because in the action of data transfer from space-time domain to frequency domain (FFT operational) increases the probability of corruption related to the board effect.

2) by increasing $\mathrm{L}$, the reading/computing time of the data set, the time of data interpretation, the machine memory involved in every computational action, the weight of intranet TLC procedure (from sensor to master station) increase.

In order to provide useful responses to detection, the system must work in real time (approx); L, from this point of view, should be short. On the other hand, an elaboration window too short can corrupt the value of $C_{\text {target }}$. The efficiency of the data processing system depends on the correct length of L coming from the balancing of requirements 1) and 2). $C$ allows to compute the best value of L: the shortest L with a stable $C_{\text {target}}$.

\section{Robustness of the Signal: An Observation about the Frequency Filters Performances}

Before the introduction of signal identification procedures based on singularity protocols [9], the increase in visibility of target signals was entrusted to the robustness techniques of frequency filtering [12] [13]. This approach is metrologi- 
cally correct when the frequency (or wavelength) of the target signal is known with a fair approximation. Indeed, this information allows the use of frequency filters HP, LP, BP consistent with the physical reality of the signal. If this information is not available, often it is obtained by information external to the FFT filter calculation protocol (conjectural frequency/frequencies, sometimes coming from very strong empirical conjectures): the result depends on a conjecture and then it is, by definition, aleatory. In the case of a detection system (e.g. anti-terrorism), the problem get worse since the wavelength of the signal (detected on time domain) is a function of the target source velocity [14] [15]; If the speed $v$ of the target.

$$
\mathrm{d} v=\mathrm{d} s / \mathrm{d} t=[\mathrm{m} / \mathrm{s}]
$$

is unknown, it is not possible to associate the detected wavelength $[f(t)]$

$$
\Lambda_{t}=\Lambda_{t}[\mathrm{~s}]
$$

to the original wavelength of the signal expressed on the space $[f(s)]$

$$
\Lambda_{s}=\Lambda_{s}[\mathrm{~m}]
$$

Detection action is made on the time domain by fixed measuring system. The result of the measure of $\Lambda$ is

$$
\Lambda=\Lambda_{\text {detected }}
$$

where $\Lambda_{\text {detected }}$ is function of the time but it is also depending to the space wavelength of the target signal; $\Lambda_{\text {detected }}$ is, in fact, a time-space function $\Lambda(t, s)$ expressed on time domain (measure domain) as

$$
\Lambda_{\text {detected }}=\frac{\Lambda_{s}}{v}=\frac{\Lambda_{s}}{s / t}=\frac{[\mathrm{m}][\mathrm{s}]}{[\mathrm{m}]}=\Lambda_{t}[\mathrm{~s}]
$$

As said, the velocity $v$ of the target source, in the operative reality, is unknown and therefore the detected signal $\Lambda(t)$ has not direct association with the target. The phenomenon of the signal wavelength variation accordingly to the target velocity is pointed out by the spectral representation of the signal (wave number spectral "migration"). In Figure 3 a sector of amplitude spectrum coming from a uw high noised magnetogram is shown. On the source data set (a uw record in very noised field) there are two signals coming from the same source (military diver with DVP). The target has two different velocities: its signals migrates from spectral O.N. 236 to spectral O.N. 206 (Figure 3) [14] [15]: the spectral delocalization of target signal is due to speed variation $\Delta v$ of the target source [16]. So to be sure to preserve the target signal the HP cut frequency must have O.N. $<206$. This frequency is too low to produce a real increase in the visibility of the wanted signal (Figure 3) [13] [14]. The variation of the speed of the source prevents the univocal definition of a possible cutting frequency. This fact makes random the evaluation of the increase in visibility of the target signal produced by the action of the standard frequency filter. 


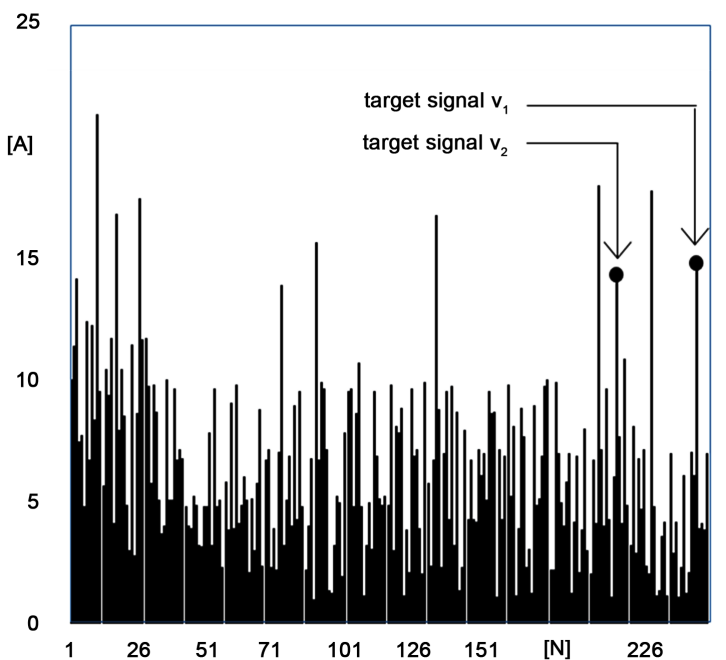

Figure 3. Example of target signal spectral $N$ migration dues to the target source velocity variations. First on source target diver $+\mathrm{DPV} V_{1}$, second one source target diver, $v_{1}>V_{2}$; spectral representation: $v_{1} \rightarrow N_{1}=236, v_{2} \rightarrow N_{2}=206$.

\section{The Signal "Visibility" in Standard and Singularity Metrologies: An Interesting Example}

With the generic term "signal visibility", we defined the capability to observe the signal inside of its spectral band or in time/space representation. Visibility is therefore a relevant feature of the signals but it is also a qualitative and subjective definition. The metrological meaning of this definition is virtually null. The qualitative parameters of the target signal visibility are (+) $\Delta A$ and $\Delta \Lambda$ against to the $A$ and $\Lambda$ of the noise signals. High (+) $\Delta A$ signal stands out, also in the time space, against the noise signals. High $\Delta \Lambda$ allows the signal to be easily isolated in the spectrum and allows to built very efficiency frequency filter. The target signal is visible when it has a graphical specific geometry with respect to the noise. In the other words if the amplitude of the target signal is low and the $\Lambda$ is within the noise band, the standard qualitative visibility approach has very low effective. In the detection action of quasi-punctiform, low amplitude and kinetic sources in high noised magnetic environment the target signal visibility is more or less zero. Moreover the variation of the speed of the source and so the spectral migration of the target signal wavenumber make subjective and totally ineffective the robustness frequency filter action. A quantitative and objective approach to controlling the visibility of each elementary signal included in a high energy band (large spectrum) is provided by the parameter $C$. $C$ connects the signal "visibility" to its amplitude and wavelength $\Lambda$. We observe now a magnetogram with a high energy content (noise) in which two target signals are present (Figure 4(a)). The qualitative observation of the magnetogram (Figure 4(a)) shows: 1) a background trend making the data series not stationary (with introduction of difficulty in the FFT calculation); 2) the corruptive presence of many man-made magnetic signals (a step and several spikes); 3) an high energy high frequency (noise band); 4) same low frequency man-made anomalies and finally 


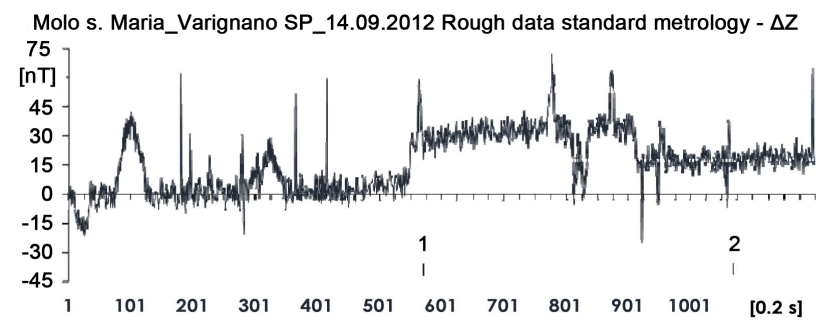

(a)

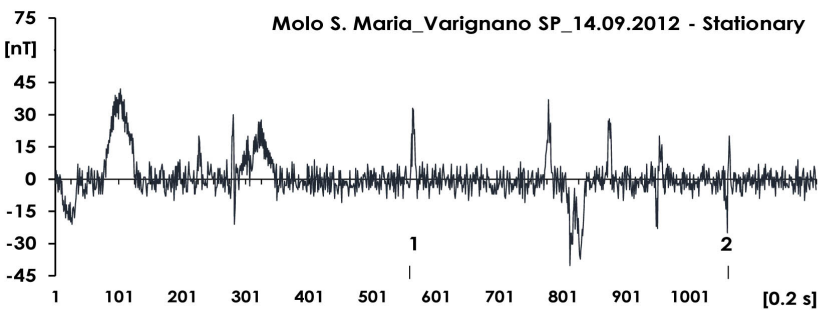

(c)

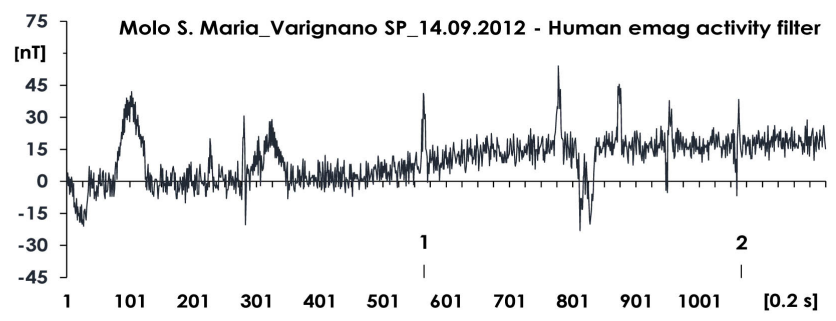

(b)

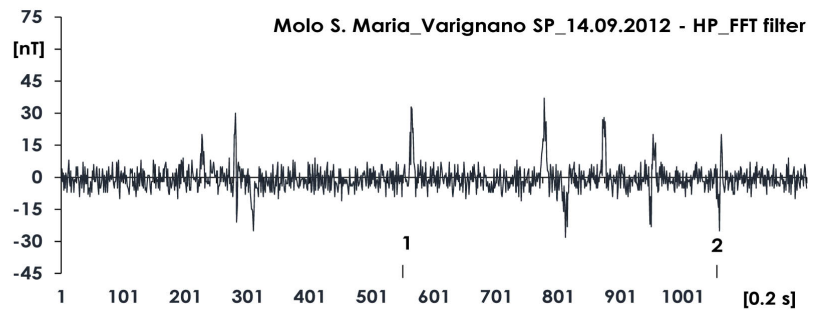

(d)

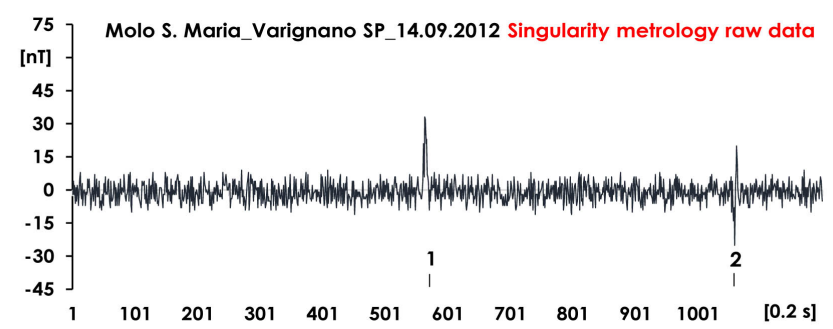

(e)

Figure 4. (a) Observed magnetic field $\Delta Z$ variation. Experiment of diver detection: Molo S. Maria, Varignano (SP) Italy, 14.09.2012., Raw data $\Delta Z[\mathrm{nT}$ ], period $T=5$ [min], sampling rate $d t=0.2$ [sec]. Metrology: direct incremental measure. 1 and 2 target signals; (b) Observed magnetic field $\Delta Z$ variation after the filter of man-made and electronic noise action. Remove action of spikes and step; (First one action to robustness standard procedure). Natural components of the earth's magnetic field plus not-classifiable artificial magnetic components; (c) Remove action of the low frequency magnetic environmental background of (Figure 4(b)) magnetogram by means linear regression filter action. Second one step of our signal robustness standard procedure; (d) HP filter action $\left(\Lambda t>15[\mathrm{~s}]\right.$ removal). In the empirical assumption $\Lambda t=15[\mathrm{~s}]$ (or more) is not compatible with $\Lambda t_{\text {target }}$ Best informative magnetogram level coming from our standard numerical robustness data manipulation. We observe 9 target class signals in the final magnetogram: target signals $=2$, not target signals $=7$ ( $\rightarrow 2$ real alarms, 7 false alarms); (e) Geomagnetic singularity measure (raw data). The magnetogram is cleaned of natural and artificial signals except the two target signals. The singularity magnetogram consists of two distinct bands of signals: high frequency and low amplitude noise band and high amplitude and low frequency informative signal band. The targets (signal 1 and 2) are reveled.

5) several (nine) "medium wavelength band" signals (this band includes the target source signals 1 and 2). In the raw data condition (measured field) the target signals 1 and 2 have visibility more or less 0.

\subsection{The $C$ Parameter in the Evaluation of Standard and Singularity Magnetometric Methods Informative Effectiveness}

The first standard signal cleaning step is the frequency filtering of the artificial signal class (man-made and instrumental electronic contributes) (Figure 4(a)). The result of this action is shows in Figure 4(b). The second step removes the background of the measured field (in the background band we note an increase 
of low frequency that makes the raw data sequence not stationary). The technique used in this action is the compensation for "linear regression" (Figure 4(c)); note: this action removes several relevant naturalistic information, but it is not useful for our scope (detection). At this point of the standard robustness procedure the increase of signal visibility is not relevant. The small difference in the target visibility introduced by this action must be linked more to calculation to calculation approximations than to the stationarization of the series (Figure $4(\mathrm{c})$ ). The third step of the signal cleaning standard procedure is the HP frequency filter. This application cuts off low frequency signals not compatible with the target signals physic characteristic (speed of the source) (Figure 4(d)). For the main purpose of this work (detection) it is not relevant to investigate the origin of these anomalies (natural or man-made); the discriminating factor is their wavelength compatible or not with the target's velocity. In conclusion, we note the standard procedure of signal robustness improving do not allow sensitive improvements of the visibility of targets 1 and 2. Let us now apply the parameter $C$ to define the informative capability value of the singularity measurement technique. The effect of the metrology of singularity is the cutting of all signals except the local singularity ones [3] [17] (marked in Figure 4(a)-(e) as 1 and 2) (Figure $4(\mathrm{e})$ ). Since the signals target are the only supersites to the action of the singularity metrology (except the HF noise not compatible with the speed of the target sources) the increase of $C 1$ and $C 2$ is high (Figure 5). This increase of $C$ quantifies the increase of the target signal visibility due to the singularity metrology.

\subsection{The Quantitative Example}

We use the coefficient $C$ (and the corresponding $e C$ ) to quantify the qualitative observations referred in Section 6.1. Figure 6 shows the graphs of $\mathrm{R}$ factor as a function of order number of the Fourier serial computation. The data shown in Figure 6 graphics are coming from a comparison test between standard magnetic measures results (and their robustness procedure) and magnetic measures of singularity one (raw data) (Figure 6).

(Table 1) Factors $C$ and $e C$ show the target signal by a diver equipped by commercial equipment $(N 18)$ have low informative capability $\left(C_{18}=0.13, e C_{18}=\right.$ 0.15 where $C=1$ is monochromatic signal) (Figure 6(a)). We now observe the

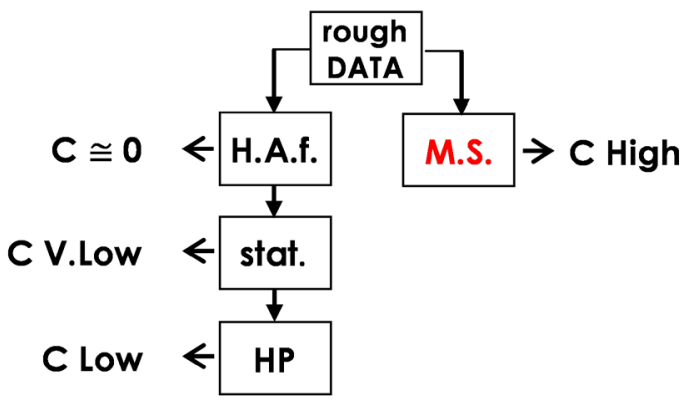

Figure 5. Qualitative view of the signal robustness computational flux (and $C$ increasing)—left and $C$ value from singularity measure (rough data)—right. 
Darsena Duca degli Abruzzi_La Spezia_27.09.2012

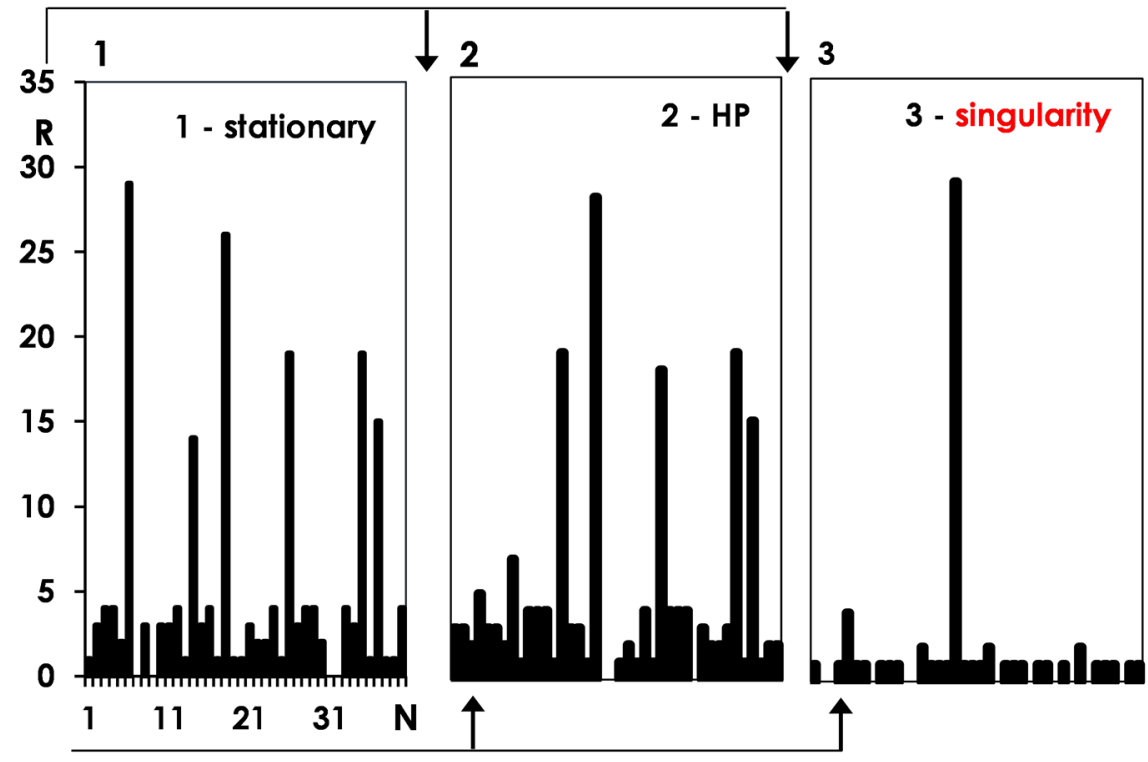

Figure 6. Quantitative graphical view N-R of $C 28$ value. 1) stationary step of standard robustness procedure, 2) HP step of standard robustness procedure, 3) metrology of singularity raw data (Table 1).

Table 1. Evolution of the informative capability $C$ and enhanced informative capability $e C$ of the $N_{18}$ signal. Field Measure: standard geomagnetic metrology data after first one and second one steps of the standard frequency filter robustness procedure (Figure 4(b), Figure 4(c)); HP: standard geomagnetic metrology data after third one step of filter robustness procedure (HP) (Figure 4(d)); Singularity: singularity geomagnetic metrology raw data (Figure $4(\mathrm{e})$ ).

\begin{tabular}{cccc}
\hline & \multicolumn{3}{c}{$N$ target $=N_{18}$} \\
\cline { 2 - 4 } & field measure & HP & Singularity \\
\hline$C$ & 0.13 & 0.15 & 0.49 \\
$e C$ & 0.15 & 0.18 & 0.73 \\
\hline
\end{tabular}

implementation of the $C$ value dues to the standard process of signal robustness. Figure 6(b) shows the result of the signal cleaning action corresponding to the final step HP filtering (cut frequency $15[\mathrm{sec}]$ ). Although this level of signal informative capability implementation is the deepest in the standard procedures of signal robustness, the informative gain is not satisfactory $(C=0.15, e C=0.18$ ) (Table 1). Also, it is not possible to estimate how much of this increment is real (due to the decrease of EP admitted to computation) or due to calculation approximations (therefore of numeric origin and non physical one). Finally we observe the result obtained from the singularity measure (Figure 6(c)): although the $C$ control is applied to a raw data set originated by singularity metrology, the increase in $C$ information capability is satisfying $(C=0.49, e C=0.73)$ (Table 1). $C$ (and $e C$ ) quantifies the much better performance of the singularity metrology respect to the standard measurement one (direct or differential field measurements). 


\section{Conclusion}

The Earth magnetic field in proximity of urban zones, critical structures, power points and power lines is a time transient one. It is characterized by high energy and very large band spectrum. This fact, in order to detect quasi-punctiform, low amplitude and kinetic sources, makes very low the effectiveness of the passive magnetometric detection technique. The metrology of singularity is a good solution for the detection of this class of signals (low visibility target signal in high time transient and very noisy field). The quantization of the effectiveness of magnetic singularity technique (and its optimization of use) is based on the evaluation of its informative capability. On the other hand, the experience shows informative capability of the small signals in hard noised field which depends more on their energy than on their amplitude. For this reason it is necessary to introduce a numerical parameter, considering this aspect. The informative capability $C$ (and its derived enhanced informative capability $e C$ ) is depending from both the amplitude and the wavelength and thus from the signals energy. The $C$ (and $e C$ ) operating use tests demonstrated the high effectiveness of this parameter in quantizing the information of the target signals. $C$ also allows to quantize the superiority of the singularity magnetic metrology approach to the standard one (direct or differential) especially in the high definition detection action. At last, $C$ contributes to the objective definition of the best length of the computing/observation windows for the counter-intrusion detection protocols. This contribute is crucial in the optimization of the weight (length) of the data sets admitted to single cycle of computation in the detection system intelligence. This fact optimizes the time of automatic alert reaction performance in the passive magnetic systems with singularity measurement technique.

\section{Acknowledgements}

This study is part of Italian Harbor/Base Protection research action. It was supported by SEGREDIFESA of the Italian Ministry of Defence under the National Military Research Plan R \& T projects La.Ma.1.0_2.0. Field trials were managed by Italian Army (IX Btg Ass. "Col Moschin") and Italian Navy (MARI.CO.DRAG. and COM.SUB.IN.); technological support by an Italian SME (SkyTech SrL-SP). Thanks to all of you.

\section{References}

[1] Faggioni, O., Pinna, E., Savelli, C. and Schreider, A.A. (1995) Geomagnetism and Age Study of Tyrrhenian Seamounts. Geophysical Journal International, 123, 915-930.

[2] Cooley, J.W. and Tukey, J.W. (1965) An Algorithm for the Machine Calculation of Complex Fourier Series. Mathematics of Computation, 19, 297-301. https://doi.org/10.1090/S0025-5718-1965-0178586-1

[3] Faggioni, O., Soldani, M., Cozzani, G. and Zunino, R. (2018) Informative Signal Analysis: Metrology of the Underwater Geomagnetic Singularities in Low-Density Ionic Solution (Sea Water). Journal of Signal and Information Processing, 9, 1-23. https://doi.org/10.4236/jsip.2018.91001 
[4] Faggioni, O., Soldani, M., Gabellone, A., Hollett, R.D. and Kessel, R.T. (2010) Undersea Harbour Defence: A New Choice in Magnetic Networks. Journal of Applied Geophysics, 72, 46-56. https://doi.org/10.1016/j.jappgeo.2010.07.001

[5] De Vuyst, A. and De Meyer, F. (1973) Spectral Analysis of Geomagnetic Data from One Station (Dourbes 1960-1970). Institut Royal Météorologique de Belgique, Bruxelles, 80, 32-41.

[6] Wax, M. and Kailath, T. (1985) Detection of Signals by Information Theoretic Criteria. IEEE Transactions on Acoustics, Speech, and Signal Processing, 33, 387-392. https://doi.org/10.1109/TASSP.1985.1164557

[7] Marks II, R.J. (2008) Hanbook of Fourier Analysis \& Its Applications. Oxford University Press, Oxford.

[8] Faggioni, O. (1986) GDS Experiments in North Italy Area: Same Quantitative Observations. Italian National Library “Vittorio Emanuele II”, Roma, Ph.D. Thesis, Consortium of Universities Genova, Modena, Torino, 23-33.

[9] Cooley, J.W., Lewis, P.A.W. and Welch, P.D. (1967) The Fast Fourier Transform Algorithm and Its Applications. Research Paper RC-1743, IBM Watson Research Centre, Yorkton Heights, NY.

[10] Decherchi, S., Leoncini, D., Gastaldo, P., Zunino, R., Faggioni, O. and Soldani, M. (2011) Computational Intelligence Methods for Underwater Magnetic-Based Protection Systems. Proceedings of IJCNN International Joint Conference on Neural Networks, San Jose, 31 July-5 August 2011, 238-245.

[11] Harris, F. (1978) On the Use of Windows for Harmonic Analysis with the Discrete Fourier Transform. Proceedings of IEEE, 66, 51-83. https://doi.org/10.1109/PROC.1978.10837

[12] Thomson, D.J. (1982) Spectrum Estimation and Harmonic Analysis. Proceedings of the IEEE, 70, 1055-1096. https://doi.org/10.1109/PROC.1982.12433

[13] Kanasewich, E.R. (1973) Time Sequence Analysis in Geophysics. The University of Alberta Press, Edmonton.

[14] NOAA, et al. (2010) NOAA Diving Manual: Diving for Science and Technology. 4th Edition, Best Publishing Company, North Palm Beach.

[15] COMSUBIN (2011) Norme per le Immersioni. Marina Militare, Roma.

[16] Gabellone, A., Faggioni, O, Soldani, M. and Guerrini, P. (2008) C.A.I.Ma.N.-Coastal Anti Intruder Magnetometers Network. Proceedings of RTO-MP-SET-130 Symposium on NATO Military Sensing, Orlando, 12-14 March 2008.

[17] Faggioni, O., Soldani, M., Leoncini, D., Gabellone, A. and Maggiani P.V. (2009) Time Domain Performances Analysis of Underwater Magnetic SIMAN Systems for Port Protection. Journal of Information Assurance and Security, 4, 538-545. 